\title{
Incidence of a first thromboembolic event in asymptomatic carriers of high-risk antiphospholipid antibody profile: a multicenter prospective study
}

\author{
Vittorio Pengo, ${ }^{1}$ Amelia Ruffatti, ${ }^{2}$ Cristina Legnani, ${ }^{3}$ Sophie Testa, ${ }^{4}$ Tiziana Fierro, ${ }^{5}$ Francesco Marongiu, ${ }^{6}$ \\ Valeria De Micheli, ${ }^{7}$ Paolo Gresele, ${ }^{5}$ Marta Tonello, ${ }^{2}$ Angelo Ghirarduzzi, ${ }^{8}$ Elisa Bison, ${ }^{1}$ Gentian Denas,${ }^{1}$ \\ Alessandra Banzato, ${ }^{1}$ Seena Padayattil Jose, ${ }^{1}$ and Sabino lliceto ${ }^{1}$
}

Departments of ${ }^{1}$ Clinical Cardiology and ${ }^{2}$ Rheumatology, University Hospital, Padova, Italy; ${ }^{3}$ Department of Angiology and Blood Coagulation Marino Golinelli, University Hospital, Bologna, Italy; ${ }^{4}$ District Hospital, Cremona, Italy; Internal and Vascular Medicine, University Hospital, Perugia, Italy; ${ }^{1}$ nternal Medicine, University Hospital, Cagliari, Italy; ${ }^{7}$ Transfusion Medicine, District Hospital, Merate, Italy; and ${ }^{8}$ Angiology Unit, Department of Internal Medicine, Santa Maria Nuova Hospital, Reggio Emilia, Italy

\begin{abstract}
Persistent antiphospholipid (aPL) antibodies are occasionally found in subjects without prior history of thromboembolic events (TEs), raising the dilemma of whether to initiate or not a primary thromboprophylaxis. A first TE is considered rare in aPL carriers, but previous studies did not consider the aPL profile nor was the test positivity confirmed in a reference laboratory. In this study, 104 subjects with high-risk aPL profile (positive lupus anticoagulant, anticardiolipin, and
\end{abstract}

anti- $\boldsymbol{\beta}_{2}$-glycoprotein I antibodies, triple positivity) confirmed in a reference laboratory, were followed up for a mean of 4.5 years. There were 25 first TEs $(5.3 \%$ per year): the cumulative incidence after 10 years was $37.1 \%(95 \%$ confidence interval $[\mathrm{Cl}], 19.9 \%-54.3 \%)$. On multivariate analysis, male sex (hazard ratio $=4.4$; 95\% $\mathrm{Cl}, 1.5-13.1, P=.007)$ and risk factors for venous thromboembolism (hazard ratio $=3.3 ; 95 \% \mathrm{Cl}, 1.3-8.5, P=.01$ ) were independent predictors for TEs. As- pirin did not significantly affect the incidence of TE. In conclusion, the occurrence of a first TE in carriers of high-risk aPL profile is considerable; it is more frequent among male subjects and in the presence of additional risk factors for venous TE. These data can help in the decision to initiate primary thromboprophylaxis in these subjects. (Blood. 2011; 118(17):4714-4718)

\section{Introduction}

It is now widely recognized that multiple positivity in tests exploring the presence of antiphospholipid (aPL) antibodies (lupus anticoagulant [LA], anticardiolipin [aCL], and anti- $\beta_{2}-$ glycoprotein I [anti- $\beta_{2} \mathrm{GPI}$ antibodies) is associated with thromboembolic events (TEs) more frequently than single test positivity. ${ }^{1-5}$ Notably, triple positivity (all 3 tests positive) is associated with thrombosis and identifies high-risk patients with aPL syndrome (APS) ${ }^{6,7}$ In this respect, the finding of a healthy person carrying aPL triple positivity poses the problem of actual risk of TEs and raises the dilemma of whether a primary prophylaxis with antithrombotic drugs should be initiated. Here, we describe a cohort of patients with triple-positive tests that were prospectively followed in Italian Thrombosis Centers, and assess TEs and their predictors.

\section{Methods}

\section{Study design and population}

This study considered patients positive for all 3 tests exploring aPL (LA, $\mathrm{aCL}$, and anti-B2GPI antibodies) on 2 occasions at least 12 weeks apart without exhibiting the clinical features of APS. Recruited subjects were seen by the center physician in an outpatient setting once a year or earlier according to their clinical status or if discharged from the hospital. No long-term anticoagulant treatment was prescribed, and all the subjects received short-term thromboprophylaxis with once-daily low molecular weight heparin in high-risk for TE scenarios (surgery or prolonged immobilization)
To confirm triple positivity for aPL and to validate the laboratory diagnosis, plasma from patients of each center was retested in a reference laboratory (Padua Thrombosis Center). Final inclusion in the cohort required confirmation of LA positivity as well as aCL and anti- $\beta_{2} \mathrm{GPI}$ antibody positivity (values in the upper 99th percentile of 40 healthy subjects) for the same isotype. The predominant isotype was considered $\mathrm{M}$ when IgM aCL value expressed as MPL units exceeded the IgG aCL value (expressed as GPL units). Patients positive for LA and aCL before anti-B2GPI ELISA was available had the test performed subsequently on the first original stored plasma. All subjects were informed about the study and gave signed consent about the treatment of their data in accordance with the Declaration of Helsinki. The study was approved by the local ethics committee of each Thrombosis Center.

\section{Data collection at laboratory diagnosis}

Centers were asked to provide demographic, laboratory, and clinical data for each patient. TEs and pregnancy morbidity before laboratory diagnosis were excluded on the basis of patient interview and available hospital records.

Information about the presence of associated autoimmune diseases (systemic lupus erythematosus [SLE] or other connective tissue disease) and the presence of additional risk factors for thrombosis was also collected. Assessed arterial risk factors were: diabetes mellitus, hypertension, hypercholesterolemia, obesity, smoking habit, and family history. Assessed venous risk factors were: oral estroprogestinic treatment, pregnancy, malignancy, family history, and thrombophilia (antithrombin, protein C, or protein S; factor V Leiden; prothrombin G20210A mutation; hyperhomocysteinemia, high factor VIII levels).
Submitted March 1, 2011; accepted June 30, 2011. Prepublished online as Blood First Edition paper, July 15, 2011; DOI 10.1182/blood-2011-03-340232.

An Inside Blood analysis of this article appears at the front of this issue.
The publication costs of this article were defrayed in part by page charge payment. Therefore, and solely to indicate this fact, this article is hereby marked "advertisement" in accordance with 18 USC section 1734.

(C) 2011 by The American Society of Hematology 


\section{Laboratory tests}

Methods and results for laboratory diagnosis refer to tests performed in the reference laboratory. Lupus anticoagulant was determined according to updated guidelines of the International Society on Thrombosis and Hemostasis $^{8}$ using a diluted Russell viper venom time kit by Instrumentation Laboratory; results of mixing tests are expressed as the ratio of diluted Russell viper venom time of 1:1 mixing of patient and normal pooled plasma, divided by diluted Russell viper venom time of pooled normal plasma (normal value, $<1.2$ ). ${ }^{9} \mathrm{aCL}$ and anti-human $\beta_{2}$ GPI antibodies were measured by home-made ELISA as previously described, ${ }^{10}$ following the proposals of the Standardization Group of the European Forum on anti-PL antibodies. ${ }^{11,12}$ aCL ELISA and anti- $\beta_{2}$ GPI ELISA were considered positive when values exceeded the cut-off value calculated using the 99th percentile of 40 normal age- and sex-matched controls. Laboratory data for ELISA tests are reported as predominant $\mathrm{IgG}$ or IgM isotypes.

\section{Outcome events}

Reports for objectively diagnosed TEs during follow-up had to include type, site, and the status of antithrombotic treatment at the time of event.

Venous thromboembolism (VTE) was diagnosed by compression ultrasonography or venography in case of deep vein thrombosis, and spiral tomography, ventilation-perfusion lung scan, or pulmonary angiography in case of pulmonary embolism. Intracerebral thrombosis was assessed by computed tomographic scanning, magnetic resonance imaging, or angiography; retinal thrombosis was diagnosed by ophthalmologic examination. Peripheral- or mesenteric-artery thrombosis was documented by arteriography or at the surgery table. Small-vessel thrombosis was evaluated by appropriate imaging study or histopathology in the absence of inflammation in the vessel wall. Acute myocardial infarction was diagnosed in the presence of a typical clinical presentation associated with typical electrocardiographic features and elevated cardiac enzymes (CK-MB or troponins I or T). Stoke/transient ischemic attack was defined according to standard definitions (transient ischemic attack was considered for analysis only if cerebral imaging confirmed cerebral ischemia). Major bleeding was defined as: fatal bleeding causing death, clinically overt bleeding associated with a fall in hemoglobin level of $\geq 20 \mathrm{~g} / \mathrm{L}$ in 24 hours, and/or requiring nonplanned transfusion of $\geq 2$ units of packed red blood cells or whole blood; intracranial bleeding (documented by imaging), retroperitoneal bleeding, intraocular bleeding causing blindness, joint hemorrhage, or need for surgery or angiographic intervention to stop hemorrhage. All other bleeding events not matching the criteria for major bleeding were considered minor bleeding. The causes of death were obtained from clinical or autopsy reports and/or death certificates.

\section{Statistics}

Descriptive statistics are reported as appropriate: categorical data are expressed as frequencies (percentage); continuous data are reported as mean SD. Kaplan-Meier survival analysis was used to determine the cumulative incidence of TEs at follow-up. Cox regression survival analysis was used to detect possible predictors of thromboembolism among the demographic factors, associated autoimmune diseases, aCL isotype, arterial and venous risk factors, and aspirin treatment at the time of event. Statistical significance was considered for $P<.05$. All analyses were performed using SPSS (Rel. 17.0.1. 2008).

\section{Results}

Of the considered 110 patients, 6 were excluded from the study (3 because of history of pregnancy loss compatible with APS and 3 for not meeting the inclusion criteria after specimen test in the reference laboratory). Demographic and clinical characteristics of 104 confirmed triple-positive carriers are depicted in Table 1. Mean age was 45 years, and $79 \%$ were female. Reasons for aPL testing were frequently an occasionally found prolonged activated partial
Table 1. Demographic and clinical characteristics

\begin{tabular}{|c|c|}
\hline Characteristics & Value \\
\hline No. & 104 \\
\hline Age, y (mean $\pm S D)$ & $45 \pm 15$ \\
\hline Female, no. (\%) & $82(79)$ \\
\hline IgG isotype, no. (\%) & $86(83)$ \\
\hline dRVVT mixing (mean $\pm \mathrm{SD}$ ) & $1.78 \pm 0.5$ \\
\hline IgG aCL, GPL units (mean \pm SD) & $102 \pm 91$ \\
\hline $\operatorname{lgG} a \beta_{2} \mathrm{GPI}$, units (mean $\pm \mathrm{SD}$ ) & $123 \pm 154$ \\
\hline \multicolumn{2}{|l|}{ IgM isotype, no. (\%) } \\
\hline dRVVT mixing & $18(17)$ \\
\hline IgM aCL, GPL units (mean \pm SD) & $1.42 \pm 0.3$ \\
\hline 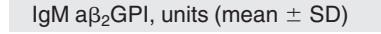 & $81 \pm 58$ \\
\hline \multicolumn{2}{|l|}{ Reason for initial testing, no. (\%) } \\
\hline Prolonged aPTT & $55(53)$ \\
\hline Autoimmune disorder & $39(37)$ \\
\hline Family history for thrombosis & $4(4)$ \\
\hline Migraine & $2(2)$ \\
\hline \multicolumn{2}{|l|}{ Autoimmune disorders, no. (\%) } \\
\hline SLE & $14(13)$ \\
\hline Other & $35(34)$ \\
\hline Risk for arterial thrombosis* no. (\%) & $34(33)$ \\
\hline Risk for venous thrombosist no. (\%) & $30(29)$ \\
\hline
\end{tabular}

dRVVT indicates diluted Russell viper venom time; and aPTT, activated partial thromboplastin time.

${ }^{*}$ Considered risk factors for arterial thrombosis: diabetes mellitus, hypertension, hypercholesterolemia, obesity, smoking habit, and family history.

†Considered risk factor for venous thrombosis: recent surgical intervention, perioperative immobilization, oral estroprogestinic treatment, pregnancy, malignancy, family history, thrombophilia, and previous VTE.

thromboplastin time or an autoimmune disorder. In the study cohort, SLE or other autoimmune diseases were present in $47 \%$ of cases, and risk factors for arterial and venous thrombosis were present in approximately one-third of subjects. IgG was the prevalent isotype in $83 \%$ of cases. During a total follow-up of 469 years, there were 25 TEs $(5.3 \%$ per year). The cumulative incidence of TEs was $9.8 \%$ (95\% confidence interval [CI], $3.7 \%-15.9 \%)$ after 2 years, $27.3 \%$ (95\% CI, $17.1 \%-37.5 \%)$ after 5 years, and $37.1 \%(95 \%$ CI, $19.9 \%-54.3 \%)$ after 10 years (Figure 1).

Details of the 25 patients who developed a TE are shown in Table 2. There were 12 arterial and 11 venous TEs, 1 thrombosis of the right atrium, and 1 renal thrombotic microangiopathy. Other

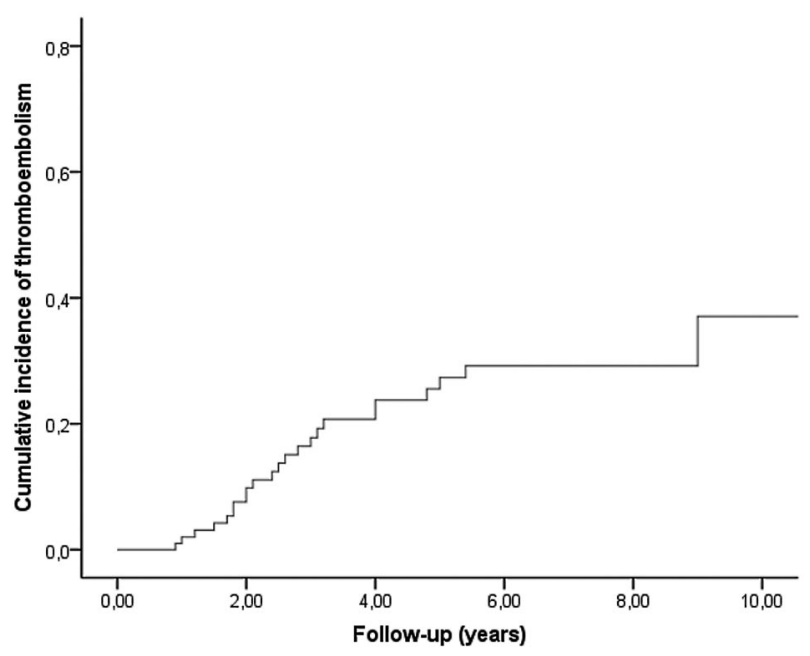

Figure 1. Cumulative incidence of TEs in the follow-up period of 104 carriers of triple positivity for aPL antibody tests. 
From www.bloodjournal.org by guest on February 2, 2016. For personal use only.

Table 2. Details of subjects affected by thromboembolism in the follow-up period

\begin{tabular}{|c|c|c|c|c|c|c|}
\hline $\begin{array}{l}\text { Patient } \\
\text { no. }\end{array}$ & Age, $y$ & Sex & Ig isotype (G or M) & Time lag, ${ }^{*} \mathbf{y}$ & Primary prophylaxis & Type of event \\
\hline 1 & 13 & Female & G & 5.4 & ASA & Stroke† \\
\hline 2 & 61 & Male & G & 0.9 & - & Stroke† \\
\hline 3 & 24 & Female & G & 18.4 & ASA & Renal thrombotic microangiopathy \\
\hline 4 & 56 & Female & G & 2.5 & - & Proximal DVT \\
\hline 5 & 54 & Male & G & 4 & ASA & Proximal DVT \\
\hline 6 & 25 & Female & G & 1.8 & - & Stroke† \\
\hline 7 & 43 & Female & G & 1.5 & - & Proximal DVT \\
\hline 8 & 30 & Female & G & 2.6 & - & PE \\
\hline 9 & 33 & Female & G & 2 & - & Stroke† \\
\hline 10 & 54 & Male & G & 4 & - & Stroke† \\
\hline 11 & 22 & Male & M & 1.2 & - & Proximal DVT + PE \\
\hline 12 & 57 & Female & G & 2.1 & - & Myocardial infarction \\
\hline 13 & 48 & Male & G & 2.4 & - & Stroke† \\
\hline 14 & 44 & Female & G & 3.1 & - & Proximal DVT + PE \\
\hline 15 & 38 & Male & G & 1.8 & - & Proximal DVT \\
\hline 16 & 50 & Male & G & 1 & - & Proximal DVT + PE \\
\hline 17 & 40 & Male & G & 3 & - & PE \\
\hline 18 & 65 & Female & G & 9 & - & TIAT \\
\hline 19 & 22 & Female & G & 1.7 & - & Right atrial thrombosis \\
\hline 20 & 57 & Female & G & 5 & ASA & TIAT \\
\hline 21 & 41 & Female & M & 11.5 & ASA & Retinal artery thrombosis \\
\hline 22 & 55 & Female & G & 4.8 & - & Proximal DVT \\
\hline 23 & 45 & Female & M & 2 & ASA & Proximal DVT \\
\hline 24 & 53 & Female & G & 3.2 & ASA & Stroke $\dagger$ \\
\hline 25 & 31 & Female & G & 2.8 & - & Stroke† \\
\hline
\end{tabular}

— indicates no primary prophylaxis; ASA, aspirin $100 \mathrm{mg} 4$ times a day; DVT, deep vein thrombosis; PE, pulmonary embolism; and TIA, transient ischemic attack.

*Time interval from laboratory finding to the thromboembolic event.

$\dagger$ All the clinical events had a pertinent area of ischemia at cerebral imaging

possible TEs not included among endpoints were superficial thrombophlebitis in 2 subjects and a case of Addison disease, the most frequent endocrine disorder in APS. Two subjects died (one of lymphoma and the other of leukemia). Pregnancy morbidity during the follow-up period occurred in 7 of the 8 females who became pregnant ( 3 were early abortion, 2 were eclampsia with premature birth, and 2 were fetal deaths).

The 25 patients with TEs were compared with the 79 controls who did not have this complication. On multivariate analysis of considered variables for TEs (Table 3), male sex (hazard ratio $=4.4 ; 95 \% \mathrm{CI}, 1.5-13.1, P=.007)$ and the presence of factors predisposing to VTE (hazard ratio $=3.3 ; 95 \% \mathrm{CI}, 1.3-8.5, P=.01$ ) were found to be independent predictors for clinical events. Aspirin prophylaxis was close to significance in reducing events on univariate analysis but did not achieve statistical significance on multivariate analysis (hazard ratio $=0.5 ; 95 \% \mathrm{CI}, 0.2-1.5, P=.2$ ). The same holds true when considering the reduction in arterial TEs.

\section{Discussion}

The clinical course in persons with high-risk aPL antibody profile (triple positivity) is not known, casting a shadow on the need for primary prophylaxis to avoid TEs. To address this issue, we evaluated a group of persons followed at Italian Thrombosis Centers, that were homogeneous in terms of aPL positivity (plasma specimens from all the subjects were tested in a reference laboratory). We previously described a group of patients with triple positivity and $\mathrm{APS}^{7}$ whose characteristics in terms of age, sex, prevalence of $\mathrm{IgG}$ isotype, and associated autoimmune diseases are similar to the group reported in this study. Results show a considerable incidence of TEs during the follow-up period with values of $9.8 \%$ after 2 years and a constant increase thereafter with an incidence of $37.1 \%$ after 10 years (annualized incidence is $5.3 \%$ ). On the basis of a limited number of retrospective and

Table 3. Risk factors for thrombosis in the follow-up period

\begin{tabular}{|c|c|c|c|c|c|c|}
\hline \multirow[b]{2}{*}{ Risk factor } & \multirow[b]{2}{*}{ Thrombosis $(\mathrm{N}=25)$} & \multirow[b]{2}{*}{ Controls $(\mathrm{N}=79)$} & \multicolumn{4}{|c|}{ Hazard ratio } \\
\hline & & & Univariate & $95 \% \mathrm{Cl}$ & Multivariate & $95 \% \mathrm{Cl}$ \\
\hline Age, y (mean $\pm S D)$ & $42.4 \pm 14.2$ & $45.3 \pm 14.7$ & 1.0 & 0.97-1.02 & - & - \\
\hline Male sex no. (\%) & $8(32)$ & $14(18)$ & 3.3 & $1.4-8.0$ & 4.4 & $1.5-13.1$ \\
\hline aCL titre, GPL units (mean $\pm \mathrm{SD}$ ) & $95 \pm 85$ & $84 \pm 91$ & 1.0 & $0.99-1.01$ & - & - \\
\hline G isotype, no. (\%) & $22(88)$ & $64(81)$ & 1.7 & $0.5-5.6$ & - & - \\
\hline Associated autoimmune diseases, no.(\%) & $10(40)$ & $39(49)$ & 0.6 & $0.2-1.1$ & - & - \\
\hline Arterial risk factors, no. (\%) & $8(32)$ & $26(33)$ & 0.9 & $0.4-2.2$ & - & - \\
\hline Venous risk factors, no. (\%) & $10(40)$ & $12(15)$ & 2.7 & $1.2-6.3$ & 3.3 & $1.3-8.5$ \\
\hline Aspirin prophylaxis, no. (\%) & $7(28)$ & $30(38)$ & 0.4 & $0.1-1.1$ & - & - \\
\hline
\end{tabular}

— indicates not significant. 


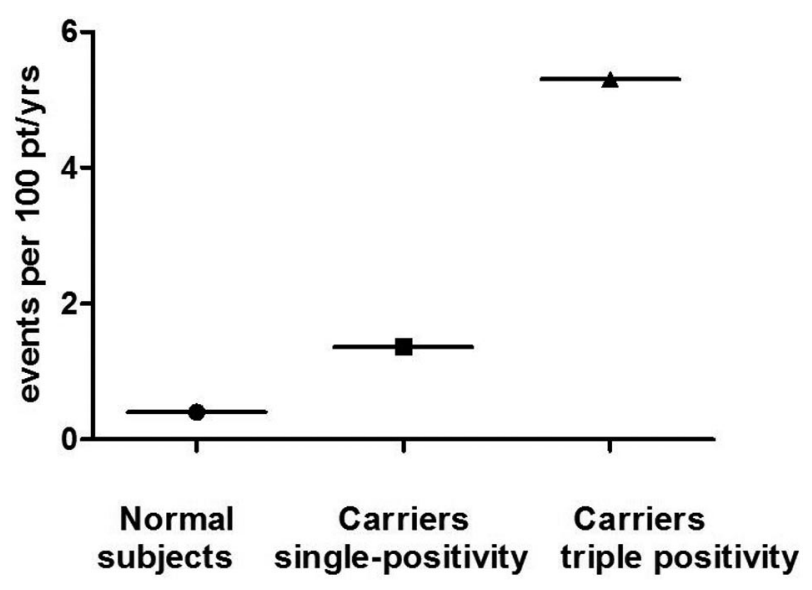

Figure 2. Average annual rates of first cardiovascular events (including VTE) in a white normal population (Ө), in single aPL-positive carriers $(\square)$, and that shown in triple-positive carriers in this study $(\mathbf{\Delta})$.

prospective studies that predominantly include SLE patients, asymptomatic aPL-positive patients have a $0 \%$ to $3.8 \%$ annual thrombosis risk. ${ }^{13}$

We report in Figure 2 the annual rate $(\sim 0.4 \%)$ of first cardiovascular event (including VTE) in normal white population at 35 to 55 years of age, ${ }^{14}$ that $(1.36 \%)$ of 125 single aPL positive carriers with a mean age of 41 years (data taken by a recently published study by our group), ${ }^{15}$ and that $(5.3 \%)$ of triple-positive carriers of this study (mean age, 45 years). Annual rate in a cohort of SLE patients half of whom had aPL was 2.9 per 100 patientyears. ${ }^{16}$ Hence, because less than half of our cohort had autoimmune disorders, the higher incidence of TEs recorded in this study may be related to the selection of high-risk aPL-positive population. Likewise, in patients with APS and triple positivity, ${ }^{7}$ TEs were equally distributed among arterial and venous circulation. Some patients in this study were treated with aspirin for primary thromboprophylaxis, behavior that did not lead to a significant reduction of events. To our knowledge, only one prospective clinical trial addresses the question of primary prophylaxis in asymptomatic aPL carriers. ${ }^{17}$ The Antiphospholipid Antibody Acetylsalicylic Acid study was a randomized, double-blind, placebocontrolled clinical trial in asymptomatic patients with persistently positive aPL comparing the efficacy of aspirin $81 \mathrm{mg}$ daily versus placebo for the prevention of thrombotic complications. The study was terminated earlier for low rate of events and showed that in patients with persistently positive aPL, aspirin was no more effective than placebo. On the other hand, other observational studies show that aspirin treatment is beneficial for primary thromboprophylaxis. ${ }^{18,19}$ In all these studies, the aPL profile of carriers is not well documented or confirmed by a reference laboratory: in the study by Giron-Gonzalez et al, ${ }^{19}$ few subjects were positive for LA, whereas most were positive in IgM aCL, an isotype showing weak or no association with TEs..$^{20,21}$ Notably, in a recent prospective study ${ }^{15}$ assessing the risk factors for a first thrombotic event in aPL antibody carriers, most of whom with autoimmune diseases, it was found that hypertension and LA positivity are independent risk factors for thrombosis, and primary thromboprophylaxis (aspirin in most cases) should probably be limited to high thromboembolic risk clinical scenarios. It is now clear that LA positivity is associated with TEs. ${ }^{22}$ However, it is mandatory to know the complete aPL profile of patients/carriers as in our hands the sole positivity for LA is not associated with thrombosis, ${ }^{6}$ or with clinical manifestations of APS. ${ }^{23}$ Similar conclusions were drawn from the Leiden thrombophilia casecontrol study, ${ }^{24}$ where LA positivity in the absence of anti- $\beta_{2}$ GPI or antiprothrombin antibodies was not associated with an augmented risk for deep vein thrombosis (odds ratio $=1.3$; 95\% CI, 0.3-6.0). It must be underlined here that, in triple-positive patients, LA potency is strong and the titer of aCL (mostly IgG) and anti- $\beta_{2}$ GPI antibodies is high. ${ }^{7,23}$

Most carriers of triple positivity in this study (64\%) were not prescribed antithrombotic prophylaxis, and the remaining were given low-dose aspirin. However, aspirin use did not result in a significant reduction either of total TEs or of the sole arterial thromboembolism. Thus, in the presence of high-risk aPL profile (triple positivity), aspirin is not effective in primary prevention and is significantly less effective than vitamin $\mathrm{K}$ antagonists in the secondary prevention. ${ }^{7}$ Searching for additional risk factors for a first TE may be crucial in decision-making. Male sex and the presence of risk factors for VTE were found to be independent risk factors for a first TE in this study. That male sex is related to a higher risk for recurrence of VTE among patients with unprovoked deep vein thrombosis is not new. ${ }^{25}$ Thus, because aspirin does not significantly affect the rate of a first TE among high-risk patients, the use of anti-vitamin $\mathrm{K}$ or new oral anticoagulants in the near future should be considered, particularly if the subject is male with other risk factors for VTE. Of the 18 patients treated with hydroxychloroquine, $2(11 \%)$ had a first TE in the follow-up period. A potential protective role of this drug is possible and may explain the risk reduction for thrombosis in patients with associated autoimmune disorders. Larger clinical trials are definitely needed to test these hypotheses.

\section{Authorship}

Contribution: V.P., A.R., and S.I. designed the research and wrote the paper; C.L., S.T., T.F., F.M., V.D., P.G., and A.G. performed research, analyzed data, and wrote the paper; M.T., E.B., and A.B. contributed analytical tools, analyzed data, and wrote the paper; and G.D. and S.P.J. analyzed data and wrote the paper.

Conflict-of-interest disclosure: The authors declare no competing financial interests.

Correspondence: Vittorio Pengo, Clinical Cardiology, Thrombosis Center, Via Giustiniani 2, 35128 Padova, Italy; e-mail: vittorio.pengo@unipd.it.

\section{References}

1. Detkov PY, Gil-Aguado A, Lavilla P, et al. Do antibodies to beta2-glycoprotein 1 contribute to the better characterization of the antiphospholipid syndrome? Lupus. 1999;8(6):430-438.

2. Lee EY, Lee CK, Lee TH, et al. Does the antibeta2-glycoprotein I antibody provide additional information in patients with thrombosis? Thromb Res. 2003;111(1):29-32.

3. Sailer T, Zoghlami C, Kurz C, et al. Anti-beta2- glycoprotein I antibodies are associated with pregnancy loss in women with the lupus anticoagulant. Thromb Haemost. 2006;95(5):796-801.

4. Zoghlami-Rintelen C, Vormittag R, Sailer T, et al. The presence of IgG antibodies against beta2glycoprotein I predicts the risk of thrombosis in patients with the lupus anticoagulant. $J$ Thromb Haemost. 2005;3(6):1160-1165.

5. Ruffatti A, Tonello M, Cavazzana A, et al. Labora- tory classification categories and pregnancy outcome in patients with primary antiphospholipid syndrome prescribed antithrombotic therapy. Thromb Res. 2009;123(3):482-487.

6. Pengo V, Biasiolo A, Pegoraro C, et al. Antibody profiles for the diagnosis of antiphospholipid syndrome. Thromb Haemost. 2005;93(6):147152.

7. Pengo V, Ruffatti A, Legnani C, et al. Clinical 
course of high risk patients diagnosed with antiphospholipid syndrome (APS). J Thromb Haemost. 2009;8(2):237-242.

8. Pengo V, Tripodi A, Reber G, et al. Subcommittee on Lupus Anticoagulant/Antiphospholipid Antibody of the Scientific and Standardisation Committee of the International Society on Thrombosis and Haemostasis: update of the guidelines for lupus anticoagulant detection. Subcommittee on Lupus Anticoagulant/Antiphospholipid Antibody of the Scientific and Standardisation Committee of the International Society on Thrombosis and Haemostasis. J Thromb Haemost. 2009;7(10):17371740.

9. Pengo V, Biasiolo A, Gresele P, et al. Survey of lupus anticoagulant diagnosis by central evaluation of positive plasma samples. J Thromb Haemost. 2007;5(5):925-930.

10. Pengo V, Biasiolo A, Fior MG. Autoimmune antiphospholipid antibodies are directed against a cryptic epitope expressed when b2-glycoprotein-I is bound to a suitable surface. Thromb Haemost. 1995;73(1):29-34.

11. Tincani A, Allegri F, Balestrieri G, et al. Minimal requirements for antiphospholipid antibodies ELISAs proposed by the European Forum on antiphospholipid antibodies. Thromb Res. 2004; 114(5):553-558.

12. Reber G, Tincani A, Sanmarco M, et al. Proposa for the measurements of anti-b2-glycoprotein I antibodies: Standardization Group of the European Forum on Antiphospholipid Antibodies. J Thromb Haemost. 2004;2(10):1860-1862.

13. Barbhaiya M, Erkan D. Primary thrombosis prophylaxis in antiphospholipid antibody-positive patients: where do we stand? Curr Rheumatol Rep. 2011;13(1):59-69.

14. Roger VL, Go AS, Lloyd-Jones DM, et al. Heart disease and stroke statistics: 2011 update. A report from the American Heart Association. Circulation. 2011;123(4):e18-e209.

15. Ruffatti A, Ross TD, Ciprian M, et al. Risk factors for a first thrombotic event in antiphospholipid antibody carriers: a prospective multicentre follow-up study. Ann Rheum Dis. 2011;70(6):10831086.

16. Mok CC, Tang SSK, To $\mathrm{CH}$, et al. Incidence and risk factors of thromboembolism systemic lupus erythematosus: a comparison of three ethnic groups. Arthritis Rheum. 2005;52(9):2774-2782.

17. Erkan D, Harrison MJ, Levy R, et al. Aspirin for primary thrombosis prevention in the antiphospholipid syndrome: a randomized, double-blind, placebo controlled trial in asymptomatic antiphospholipid antibody-positive individuals. Arthritis Rheum. 2007;56(7):2382-2391.

18. Hereng $T$, Lambert M, Hachulla E, et al. Influence of aspirin on the clinical outcomes of 103 antiphospholipid phospholipid antibodies-positive patients. Lupus. 2008;17(1):11-15.
19. Giron-Gonzalez JA, Garcia del Rio E, Rodriguez C, et al. Antiphospholipid syndrome and asymptomatic carriers of antiphospholipid antibody: prospective analysis of 404 individuals. J Rheumatol. 2004;31(8): 1560-1567.

20. Galli M, Borrelli G, Jacobsen EM, et al. Clinical significance of different antiphospholipid antibodies in the WAPS (warfarin in the antiphospholipid syndrome) study. Blood. 2007; 110(4):1178-1183.

21. Pengo V. A contribution to the debate on the laboratory criteria that define the antiphospholipid syndrome. J Thromb Haemost. 2008;6(6):1-2.

22. Galli M, Luciani D, Bertolini G, et al. Lupus anticoagulants are stronger risk factors for thrombosis than anticardiolipin antibodies in the antiphospholipid syndrome: a systematic review of the literature. Blood. 2003;101(5):1827-1832.

23. Pengo V, Biasiolo A, Gresele P, et al. A comparison of lupus anticoagulant-positive patients with clinical picture of antiphospholipid syndrome and those without. Arterioscler Thromb Vasc Biol. 2007;27(12):e309-e310.

24. de Groot PG, Lutters B, Derksen RH, et al. Lupus anticoagulants and the risk of a first episode of deep venous thrombosis. J Thromb Haemost. 2005;3(9):1993-1997.

25. McRae S, Tran H, Schulman S, et al. Effect of patient's sex on risk of recurrent venous thromboembolism: a meta-analysis. Lancet. 2006; 368(9533):371-378 


\section{Incidence of a first thromboembolic event in asymptomatic carriers of high-risk antiphospholipid antibody profile: a multicenter prospective study}

Vittorio Pengo, Amelia Ruffatti, Cristina Legnani, Sophie Testa, Tiziana Fierro, Francesco Marongiu, Valeria De Micheli, Paolo Gresele, Marta Tonello, Angelo Ghirarduzzi, Elisa Bison, Gentian Denas, Alessandra Banzato, Seena Padayattil Jose and Sabino lliceto

Updated information and services can be found at:

http://www.bloodjournal.org/content/118/17/4714.full.html

Articles on similar topics can be found in the following Blood collections

Clinical Trials and Observations (4250 articles)

Thrombosis and Hemostasis (930 articles)

Information about reproducing this article in parts or in its entirety may be found online at:

http://www.bloodjournal.org/site/misc/rights.xhtml\#repub_requests

Information about ordering reprints may be found online at:

http://www.bloodjournal.org/site/misc/rights.xhtml\#reprints

Information about subscriptions and ASH membership may be found online at:

http://www.bloodjournal.org/site/subscriptions/index.xhtml 